\title{
Probe confocal laser endomicroscopy in the therapeutic endoscopic management of Barrett's dysplasia
}

\author{
Fabrice Caillola , Sebastien Godata, Flora Poizat $^{\mathrm{b}}$, Aurélie Auttret ${ }^{c}$, Christian Pesentia, Erwan Bories ${ }^{a}$, \\ Jean Phillipe Ratone ${ }^{a}$, Marc Giovanninia
}

Paoli Calmettes Institute, Marseille, France

\section{Abstract}

\section{Introduction}

Barrett's esophagus (BE) involves the replacement of the squamous mucosa with specialized intestinal metaplasia that can evolve in the following sequence: metaplasia, low-grade

${ }^{a}$ Endoscopy Unit (Fabrice Caillol, Sebastien Godat, Christian Pesenti, Ewran Bories, Jean Phillipe Ratone, Marc Giovannini); ${ }^{\text {Pathology }}$ Unit (Flora Poizat); 'Statistics Unit (Aurélie Auttret), Paoli Calmettes Institute, Marseille, France

Conflict of Interest: None

Correspondence to: Fabrice Caillol, MD, Endoscopy Unit, Paoli Calmettes Institute, 232 bd Ste Marguerite, 13009, Marseille, France, e-mail: fcaillol@free.fr

Received 06 October 2016; accepted 28 February 2017; published online 24 March 2017

DOI: https://doi.org/10.20524/aog.2017.0138 dysplasia (LGD), high-grade dysplasia (HGD), and esophageal adenocarcinoma (EAC) [1]. Endoscopic management depends on the histological stage of $\mathrm{BE}$ and includes the following: follow up; endotherapy with thermal ablation; and endoscopic resection (ER), either piecemeal with mainly cap-assisted endoscopic mucosal resection (EMR), monobloc with the use of submucosal dissection (ESD), or surgical management in cases with invasive lesions [2]. The therapeutic management of BE depends on biopsy results, and we know that biopsies are unreliable in $20-75 \%$ of cases [3,4]. Consequently, several techniques have been introduced to improve the diagnosis of the histological stage of BE. With improvements in imaging (i.e. high-definition [HD] vision, electronic coloration, and zoom) endoscopy permits the detection of dysplasia in nearly $90 \%$ of cases, but this has only been demonstrated in a handful of studies by teams of Asian experts who compared the results with those of biopsies [5]. In parallel, confocal laser endomicroscopy (CLE) allows a histological view of the 
mucosa and has been demonstrated to result in an increase in HGD detection in BE [6-9].

In the present study, CLE was used to detect dysplasia. Patients were referred to our center for the therapeutic management of dysplasia in BE, and the aim of our study was to test whether CLE could be used to confirm the accuracy of the diagnoses based on biopsy, and, when biopsy proved inaccurate, permit changes in therapeutic management in real time.

\section{Patients and methods}

This was a retrospective study based on a prospective registry of patients referred for the management of $\mathrm{BE}$ associated with at least LGD on previous biopsies, i.e., all patients had at least LGD on the biopsies. Inclusion criteria were: presence of macroscopic BE; dysplasia associated with $\mathrm{BE}$ on pre-resection biopsy; a CLE examination of visible lesions in BE; and ER of the examined areas because of HGD or suspicious lesions with LGD on the pre-resection biopsies. The lack of a biopsy, CLE or ER was an exclusion criterion. The macroscopic aspects of the $\mathrm{BE}$ and the examined areas had to be described as elevated or non-elevated lesions.

The pre-resection biopsy followed the Seattle protocol associated with targeted biopsies in suspicious areas. The biopsies were read by pathologists from our hospital or from an external center experienced in gastrointestinal diseases. The biopsy results were known before the pCLE and ER, because the aim of the study was to determine the influence of pCLE examination before treatment. Only the worst histology was taken into account.

CLE examinations (pCLE: probe CLE) were performed on visible lesions with the Gastroflex ${ }^{\circledR}$ probe from the Maunakea company, Paris, France. A cap was inserted at the top of the endoscope to limit its movements, which made the pCLE examinations easier. After the injection of fluorescein, the pCLE examination was performed in real time in each case, based on the consensus of 2 experienced endoscopists who performed all of the ERs. CLE criteria were those described by Wallace et al [10], with the addition of the description of LGD (Table 1). LGD was defined by the lack of HGD/EAC and thickness and moderate irregularity of the glands. The examined area was unmarked so as not to hamper the ER and pathological readings.

ER was performed either using either a piecemeal (single snare or cap-assisted EMR with a Duette System ${ }^{\circledR}$ from Cook Medical) or an en bloc method (ESD). The ERs were at least semi-circumferential, and thus sufficiently large to ensure that the target area was resected.

We considered 4 potential diagnoses: normal or inflammatory mucosa, metaplasia (i.e., BE), LGD, and HGD/EAC. The primary endpoints were the sensitivities, specificities and accuracies for the detection of HGD/EAC by pCLE and pre-resection biopsies, compared with those of ER histology. The secondary endpoints were the sensitivities, specificities, and accuracies for the detection of LGD and BE without dysplasia by pCLE.
Table 1 Miami criteria for Barrett's esophagus (BE) and dysplasia and added criteria for low-grade dysplasia (LGD)

\begin{tabular}{lll}
\hline $\begin{array}{l}\text { BE without } \\
\text { dysplasia }\end{array}$ & BE with LGD & BE with HGD/EAC \\
\hline $\begin{array}{l}\text { Uniform villiform } \\
\text { architecture }\end{array}$ & $\begin{array}{l}\text { Regular thickened } \\
\text { epithelial borders }\end{array}$ & $\begin{array}{l}\text { Disorganized/loss of } \\
\text { villiform structures } \\
\text { and crypts }\end{array}$ \\
Columnar cells & $\begin{array}{l}\text { Thickened } \\
\text { epithelial borders }\end{array}$ & $\begin{array}{l}\text { Dark, irregularly } \\
\text { thickened epithelial } \\
\text { borders }\end{array}$ \\
Dark goblet cells & Dilated vessels & $\begin{array}{l}\text { Dilated irregular } \\
\text { vessels }\end{array}$ \\
\hline
\end{tabular}

HGD, high-grade dysplasia; EAC, esophageal adenocarcinoma

\section{Statistical analysis}

The statistical analyses were performed using SAS version 9.3 (SAS Institute, Cary, NC, USA). The baseline characteristics were summarized using the following descriptive statistics: medians and ranges for continuous variables; numbers and percentages for categorical variables. The aims of this study were to evaluate the abilities of the confocal and biopsy techniques to determine the histologies (EAC, HGD, and BE). To this end, the concordances, sensitivities and specificities were calculated and then compared using non-parametric Cochran's Q tests to determine whether there were significant differences in the diagnostic abilities of these two techniques.

\section{Results}

Data were collected from 35 examinations conducted during 35 endoscopic procedures in 31 patients from January 2013 to January 2015. The mean length of BEs was C2.77 [1-9] M4.03 [1-9]. The lesions were elevated (nodular) in 17 cases and non-elevated (or depressed) in 3 cases. There were 2 cases of post-therapeutic stenosis. In 13 cases, the lesions were only irregularities of the mucosa that were made visible thanks to the HD endoscopic vision. The ERs were semi-circumferential in 15 cases and circumferential in 20 cases. The ERs were performed with a single snare in 6 cases, with cap-assisted EMR in 23 cases, and with ESD in 6 cases. The histological results from the resections were normal/inflammatory in 3 cases, BE in 8 cases, LGD in 10 cases, and HGD/EAC in 14 cases. No complications were observed.

Correct diagnoses were made in $71 \%(25 / 35)$ of the cases by pCLE and in 43\% (15/35) of the cases by pre-resection biopsy. The sensitivity, specificity, and accuracy for the detection of $\mathrm{HGD} / \mathrm{EAC}$ was $92.9 \%, 71.4 \%$ and $80 \%$ for pCLE, and $78.6 \%, 61.9 \%$, and $68.6 \%$ for histological biopsy, respectively. However, the differences in favor of pCLE were not statistically significant ( $\mathrm{P}=0.25$; Table 2$)$.

Fourteen patients had HGD/EAC after ER. One case of HGD/EAC was missed by pCLE and 3 cases were missed by the pre-resection biopsies. In the cases of agreement between the pre-resection biopsies and pCLE (21/35), the sensitivity, 
specificity and accuracy for HGD/EAC (12 HGD/EAC cases and 9 non-HGD/EAC cases) were $92 \%, 89 \%$, and $90 \%$, respectively.

Thirteen patients exhibited irregularities of the mucosa without elevated or depressed lesions (2 HGD/EAC cases and 11 non-HGD/EAC cases). Among these 13 patients, pCLE led to positive redirections of therapy in $70 \%(9 / 13)$ of the cases. There was one misdiagnosis (8\%), but no cases of HGD/ EAC were missed by CLE. In these cases, pCLE exhibited a sensitivity, specificity, and accuracy for the detection of HGD/EAC of $100 \%, 82 \%$, and $85 \%$, respectively, compared with $55 \%, 50 \%$ and $54 \%$, respectively, for the pre-resection biopsies ( $\mathrm{P}=0.22$; Table 3$)$. Among these 13 patients, 6 patients exhibited HGD/EAC on pre-resection biopsy. In one case, the pCLE and ER confirmed this finding, and in the other 5 cases, pCLE and ER revealed no HGD/EAC. Six other patients had LGD on the pre-resection biopsies, including 3 cases in which pCLE revealed HGD that was confirmed by ER in one case and invalidated in the 2 other cases (in one case the ER invalidated the pre-resection biopsy and pCLE results). In 2 cases, ER confirmed the pCLE findings of no dysplasia, and in 1 case, the ER confirmed pCLE validation of LGD. The last patient had BE without metaplasia on the pre-resection biopsy, and pCLE found LGD that was confirmed by ER. In this subgroup, we were able to avoid ERs in four cases (i.e. the HGD on the biopsies was not validated by the CLE), and in one case, we performed ER instead of applying ablative therapy.

The macroscopic visualizations of 14 elevated lesions (9 HGD/EAC and 5 non-HGD/EAC) resulted in a sensitivity, specificity, and accuracy for the detection of HGD/EAC of $64.7 \%, 81.3 \%$, and $72.7 \%$, respectively, and these values were significantly better than those for the non-visualized lesions $(\mathrm{P}=0.008)$. For these elevated lesions, pCLE resulted in a sensitivity, specificity, and accuracy of $100 \%, 40 \%$, and $78.6 \%$, respectively, whereas biopsy resulted in values of $89 \%, 60 \%$, and $78.6 \%$, respectively $(\mathrm{P}=0.2)$.

Regarding the detection of LGD, pCLE had a sensitivity, specificity, and accuracy of $70 \%, 84 \%$, and $80 \%$, respectively, whereas the pre-resection biopsies resulted in values of $40 \%$, $60 \%$, and $54.3 \%$, respectively $(\mathrm{P}=0.5)$. Regarding the detection of BE without dysplasia, pCLE had a sensitivity, specificity, and accuracy of $62.5 \%, 100 \%$, and $91.4 \%$, respectively, whereas the pre-resection biopsies resulted in values of $0 \%, 96 \%$, and $74 \%$, respectively $(\mathrm{P}=0.02)$.

Regarding the 2 cases of stenosis, in one case, LGD was detected by pre-resection biopsy and pCLE and confirmed by ER. In the other case, pre-resection biopsy found the LGD, pCLE found the HGD/EAC, and ER invalidated these results with a diagnosis of BE without metaplasia (Figs. 1-3).

\section{Discussion}

Our study is an evaluation of the diagnostic efficacies of preresection biopsy and pCLE compared with histology obtained by ER and of the potential influence of pCLE in the management

Table 2 Sensitivity, specificity and accuracy of biopsies and CLE for HGD/EAC

\begin{tabular}{|c|c|c|c|c|c|c|}
\hline \multirow{2}{*}{$\begin{array}{l}\text { Diagnostic } \\
\text { method }\end{array}$} & \multicolumn{2}{|c|}{ Endoscopic resection } & \multirow[t]{2}{*}{ Sensitivity (\%) } & \multirow[t]{2}{*}{ Specificity (\%) } & \multirow[t]{2}{*}{ Accuracy (\%) } & \\
\hline & HGD/EAC & No HGD/EAC & & & & \\
\hline \multicolumn{7}{|l|}{ CLE diagnosis } \\
\hline HGD/EAC & 13 & 5 & 93 & 74 & 82 & $\mathrm{P}=0.25$ \\
\hline no HGD/EAC & 1 & 14 & & & & \\
\hline \multicolumn{7}{|l|}{ Biopsy diagnosis } \\
\hline HGD/EAC & 11 & 8 & 79 & 58 & 67 & \\
\hline no HGD/EAC & 3 & 11 & & & & \\
\hline
\end{tabular}

CLE, confocal laser endomicroscopy; HGD, high-grade dysplasia; EAC, esophageal adenocarcinoma

Table 3 Sensitivity, specificity and accuracy of CLE and biopsies with non-elevated lesions (13 patients)

\begin{tabular}{|c|c|c|c|c|c|c|}
\hline \multirow{2}{*}{$\begin{array}{l}\text { Diagnostic } \\
\text { method }\end{array}$} & \multicolumn{2}{|c|}{ Endoscopic resection } & \multirow[t]{2}{*}{ Sensitivity (\%) } & \multirow[t]{2}{*}{ Specificity (\%) } & \multirow[t]{2}{*}{ Accuracy (\%) } & \\
\hline & HGD/EAC & no HGD/EAC & & & & \\
\hline \multicolumn{7}{|l|}{ CLE diagnosis } \\
\hline HGD/EAC & 2 & 2 & 100 & 82 & 85 & $\mathrm{P}=0.22$ \\
\hline no HGD/EAC & 0 & 9 & & & & \\
\hline \multicolumn{7}{|l|}{ Biopsy diagnosis } \\
\hline HGD/EAC & 1 & 5 & 50 & 55 & 55 & \\
\hline no HGD/EAC & 1 & 6 & & & & \\
\hline
\end{tabular}

CLE, confocal laser endomicroscopy; HGD, high-grade dysplasia; EAC, esophageal adenocarcinoma 


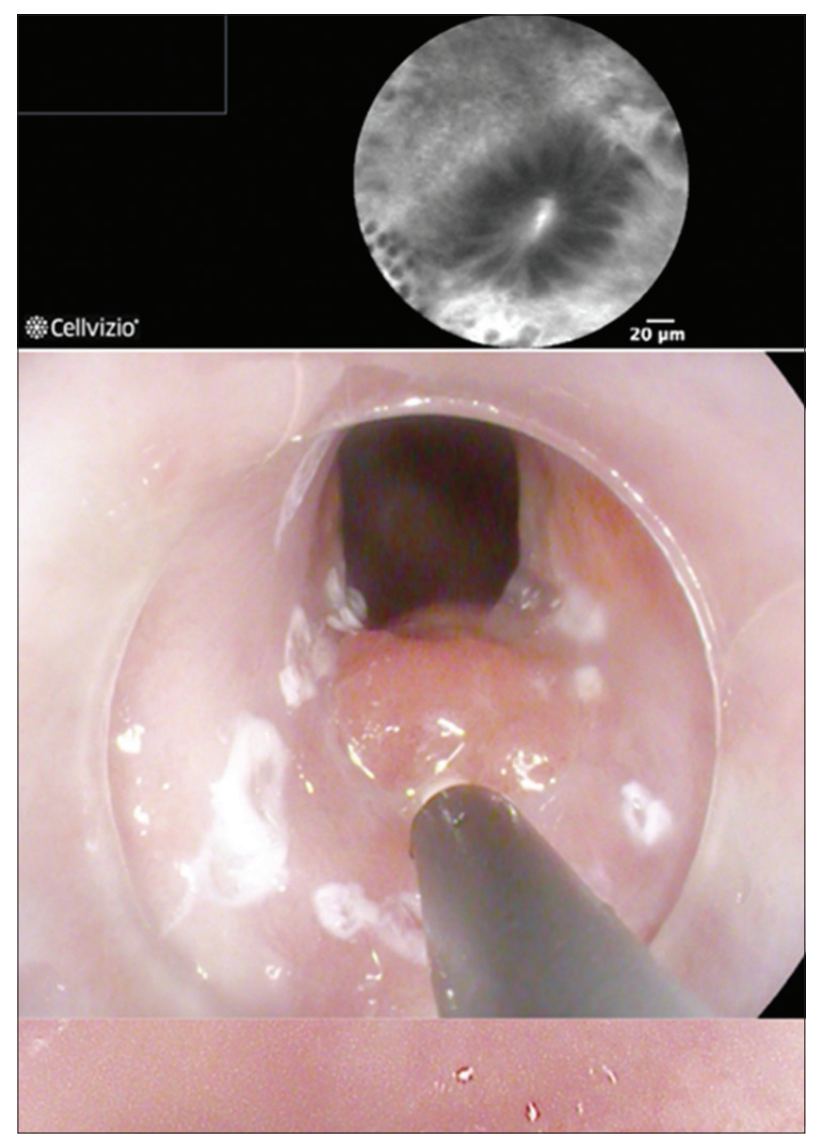

Figure 1 Elevated lesion with HGD on pre-resection biopsies, metaplasia with CLE confirmed by ER

$H G D$, high-grade dysplasia; CLE, confocal laser endomicroscopy; $E R$, endoscopic resection

of Barrett's dysplasia. We found greater histological reliability with pCLE than with pre-resection biopsies, although this difference was not statistically significant. Despite some limitations, this study has two strong points. First, benchmark histology can be obtained by ER and not by unreliable biopsies. Second, pCLE is potentially useful in the characterization of flat lesions found in $\mathrm{BE}$, the incidence of which seems to increase with HD endoscopy. Thus, HD endoscopy offers the possibility of applying treatment in real time without waiting for new pathological findings.

Most authors use pCLE to target biopsies [6,7]. In the meta-analysis conducted by Gupta et al [11], which included 8 studies with 345 patients, a per-lesion analysis revealed a pooled sensitivity of $68 \%$ and a pooled specificity of $88 \%$, while a per-patient analysis revealed a pooled sensitivity of $86 \%$ and a pooled specificity of $83 \%$. All of the included series used biopsies for the benchmark histologies. In a study by Canto et al (which was not included in the above-mentioned metaanalysis), pCLE was analyzed for its value in the detection of HGD [8]. The benchmark histologies for the 94 patients who were studied with pCLE were ER for 22 patients and biopsies for 72 patients, but the results were combined. This group yielded very good results for the detection of HGD. A per-biopsy analysis of 192 patients found the BE neoplasia

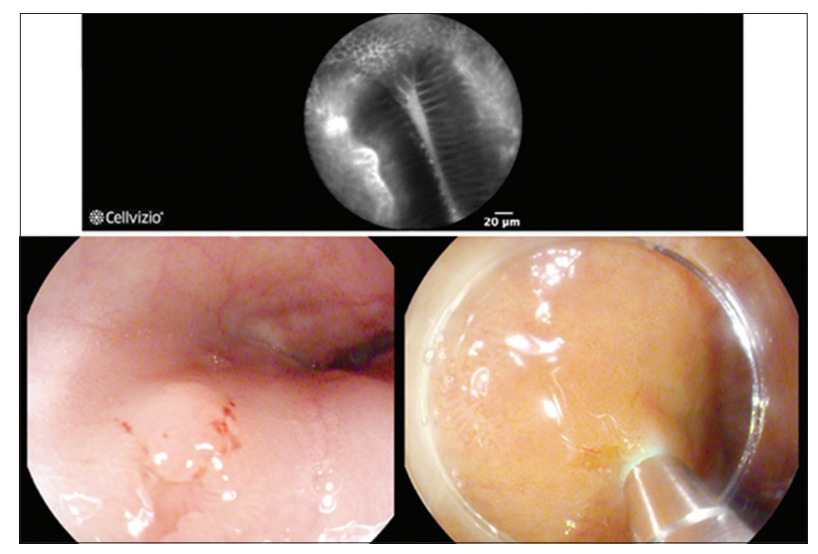

Figure 2 Irregularities of mucosa. HGD on pre-resection biopsy, LGD on PCLE, LGD after ER

$H G D$, high-grade dysplasia; LGD, low-grade dysplasia; $p C L E$, probe confocal laser endomicroscopy; ER, endoscopic resection
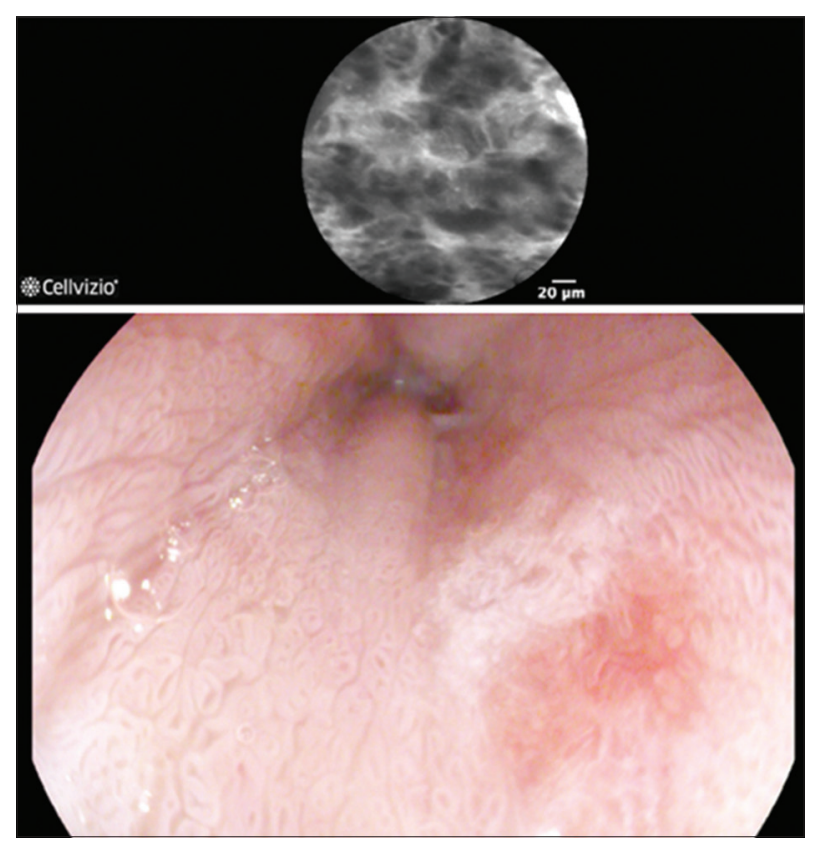

Figure 3 Irregularities of mucosa: LGD on pre-resection biopsy, HGD/ EAC on PCLE, EAC $\mathrm{m} 2$ on ER

$L G D$, low-grade dysplasia; HGD, high-grade dysplasia; EAC, esophageal adenocarcinoma; $E R$, endoscopic resection

rate to be significantly higher when pCLE was used (86\% compared to $10 \%$ with white-light endoscopy [WLE]). pCLE also significantly increased the negative predictive value (NPV) from $94 \%$ with WLE to $98 \%$. However, the specificity was lower in the pCLE group (93\% vs. 99\%), but the accuracies were similar (92\% and 93\%). A per-patient analysis revealed that, with the addition of pCLE, the sensitivity increased significantly from $40 \%$ to $95 \%$, the NPV increased significantly from $90 \%$ to $98 \%$, while the specificity decreased (from $98 \%$ to $92 \%$ ). As Gupta et al reminded us, the Preservation and Incorporation of Valuable Endoscopic Innovation that was implemented in 2012 by the American Society of Gastroenterology states that 
to replace the Seattle protocol, a technique should have a perpatient sensitivity of at least $90 \%$, an NPV of at least $98 \%$, and a specificity of at least $80 \%$ for the detection of HGD/EAC. The meta-analysis did not obtain these results, although the study of Canto et al did.

All of these studies relied on biopsy results. However, as the study by Wani et al demonstrated, ER results contradict biopsy results in $30 \%$ of cases [12]. The goal of our study was to compare pCLE results with more reliable histology results.

One limitation of our study lies in the pathological readings of the biopsies. While all pre-resection biopsies were subjected to a double reading, not all of the readings were performed in our center. We are aware of difficulties of diagnosing dysplasia, particularly LGD, in BE [13]. Because of the admitted difficulty of reading pathological findings in $\mathrm{BE}$, the confirmation of pathological findings by 2 specialized gastrointestinal pathologists is recommended [14]. Nevertheless, the term "gastrointestinal pathologist" is not well defined. Moreover, there are only very limited data regarding the interobserver variability between general and gastrointestinal pathologists [15].

In contrast, we have some data that demonstrate low interobserver agreement in diagnoses between gastrointestinal pathologists. Montgomery et al [13] demonstrated poor agreements for LGD $(\mathrm{K}=0.65)$ and indefinite dysplasia $(\mathrm{K}=0.15)$ among these pathologists. We also know that the risk of progression from LGD to carcinoma ranges from 2-40\% depending on the study. This variability is probably due to the poor interobserver reproducibility [15]. In a study that included 168 patients, pre-resection biopsies were reviewed by 7 gastrointestinal pathologists, after histologic consensuses had been reached for 5 of the 168 patients. Agreement among all 7 pathologists was observed in only $12 / 163(7.4 \%)$ of the cases. In 10 of these 12 cases, invasive carcinomas could not be excluded. The overall kappa score for all diagnoses defined in this study (i.e. HGD, HGD or intramucosal carcinoma, intramucosal adenocarcinoma, and submucosal adenocarcinoma) was fair $(\mathrm{K}=0.30)$, the highest score (for HGD) was moderate (0.47), and the lowest score (for submucosal carcinoma) was very poor (0.14) [16]. Consequently, it can be concluded that pathological readings by gastrointestinal pathologists do not resolve controversies related to the readings of pathological biopsies in BE.

We should remind the reader that there is discordance between pre-resection biopsy and EMR-based pathologies. EMR can be considered as the gold standard relative to pre-resection biopsy, given its better interobserver concordance $[17,18]$. Recently, another publication found that the accuracy of pre-resection biopsies compared with that of ER pathology in 142 cases was only 61\% (downgrades in $16 \%$ and upgrades in $23 \%$ of cases) after the gastrointestinal pathologists' readings. Some cases exhibited no dysplasia on the pathology resections (6 cases), which was consistent with the results of studies by others and our own study [19].

In the therapeutic management of BE, biopsies are essential for selecting the appropriate treatment, but several studies have demonstrated that biopsies lack reliability. In a series published in 2011, 55 patients (58\%) who were referred for T1 lesions with HGD/intramucosal cancer on biopsy had an invasive Sm2 lesion [20]. In another series, while biopsies revealed HGD, 9 patients (78\%) with visible lesions had EAC on ER pathology, and the value for patients with no visible lesions was $32 \%$ $(7 / 22)$ [21]. In a surgical series, the authors found that $12 \%$ $(3 / 25)$ of the cases had EAC with submucosal invasion without endoscopic lesions [22]. Regarding previous endoscopic studies involving evaluations of pre-resection biopsies and the pathological results of ERs, we found ER without dysplasia or without BE in 4/75 patients in the study by Moss et al [23], 7/19 patients in the study by Ahmad et al [24], 4/25 patients in the study by Nijhawan et al [25], and 2/12 patients in the study by Seewald et al [26]. All of these studies were performed after pre-resection biopsies with HGD. It is not so surprising that, with the improvement of the scope, we found more lesions; however, they were not systematic with dysplasia and consequently needed to be better characterized.

The Seattle protocol is used to minimize sampling errors and not only requires many biopsies of suspicious lesions, but also a very high number of systematic biopsies. Indeed, the number is so high that the protocol is not well applied. The protocol is probably not very efficient in the detection of dysplasia $[27,28]$.

With improvements in endoscopic imaging, several authors have attempted to decrease the numbers of biopsies required. HD imaging with targeted biopsies should allow a level of dysplasia detection that is equivalent to that of the Seattle protocol [29]. Likewise, targeted biopsies with electronic enhancement allow for significantly better detection of HGD than white-light HD endoscopy using the Seattle protocol (21 vs. $30 \%$ ) [30].

By improving detection, HD endoscopy also increases the number of lesions observed by the endoscopist, which, in this context, explains the increase in ER in the English Registry of Radiofrequency Ablation (RFA). This registry revealed an increase in ER from $48 \%$ to $60 \%$ between 2008 and 2013 and a parallel decrease in relapse rates from $13 \%$ to $2 \%$ [31]. Thus, the problem arising in therapeutic management is one of characterizing lesions that are undetected by biopsy. Although the effect was not significant in our study, pCLE could help us improve the in vivo characterization of histology. In this respect, our subgroup of patients without elevated or depressed lesions is important. In this subgroup, pCLE corrected the diagnoses in $70 \%$ of cases. In other words, using pCLE, a patient who is referred for RFA with LGD can be upgraded to ER based on the detection of HGD/EAC in real time.

Our study had some limitations. Major limitations are the retrospective design and the small number of patients. This small sample size could explain why our results did not reach significance, despite the trend toward a difference (i.e., 92.9\% and $78.6 \%$ sensitivities for the detection of HGD with pCLE and biopsy, respectively). Another limitation is that the endoscopists were not blinded to the pre-resection biopsy results. However, it is common practice for endoscopists to know the pathology results before ER, and we sought to maintain this condition in our study. With this non-blinded method, the decision for ER included an account of the pre- 
resection biopsy results. This situation is not the case for metaplasia, which makes the evaluation of PCLE as a tool for the diagnosis of metaplasia difficult and thus biased the selected population. Another limitation could be the concordance between the area targeted and the resection area. As we noted in the Methods section, we performed extended resections to ensure that the targeted area was resected, and we only accounted for the worst pathologies. We were able to mark the targeted area; however, pathological readings with coagulated target areas would also be a limitation. Another potential issue is, that in our study, the gastroenterologists also read the pCLEs. Because they provide a microscopic view of cells and vessels that is very similar to classical pathology, pCLE results should arguably be read by a pathologist, and this procedure is one potential means to improve the technique. Regarding the technique itself, one limitation is the inability of pCLE to determine the depth invasion and the consequent inability to differentiate between HGD and EAC. Overcoming this issue will be an important step for determining the management of $\mathrm{BE}$. The technique is also limited to target procedures, and not all BE lesions can be examined. This issue could represent another limitation. Finally, we should discuss our criteria for LGD. The diagnosis of LGD is difficult for pathologists because of the lack of clear criteria. We must acknowledge that this shortcoming also affects diagnosis with CLE. However, the main indication and advantage we found for the use of CLE in our study was the detection of HGD after LGD had been diagnosed based on classical pathology.

In conclusion, it is still difficult to have confidence in the pre-therapeutic histology in the management of BE. However, pCLE could help to determine the histology before ET endoscopic treatment in the endoscopic management of BE. In the absence of visible lesions, pCLE appears to correctly

\section{Summary Box}

\section{What is already known:}

- Endoscopic treatment is the first-line therapy of dysplasia in Barrett's esophagus

- Endoscopic treatment is based on pre-endoscopic biopsy

- Biopsies for pre-endoscopic treatment are not reliable

\section{What the new findings are:}

- Confocal laser endomicroscopy (CLE) is a relatively new technique used for gastrointestinal disease

- CLE assumes a comparison with pre-endoscopic biopsies

- CLE can positively change therapeutic endoscopic treatment, in real time, avoiding repeat diagnostic endoscopy diagnose close to $70 \%$ of cases. pCLE could be applied before thermal ablation in the therapeutic management of LGD to detect HGD/EAC, which would provide greater scope for altering the path of ongoing therapy. Technical progress still needs to be made, but real-time decision making using pCLE could aid in the management of BE.

\section{References}

1. Sampliner RE; Practice Parameters Committee of the American College of Gastroenterology. Updated guidelines for the diagnosis, surveillance, and therapy of Barrett's esophagus. Am J Gastroenterol 2002;97:1888-1895.

2. Pasha SF, Acosta RD, Chandrasekhara V, et al; ASGE Standards of Practice Committee. The role of endoscopy in the evaluation and management of dysphagia. Gastrointest Endosc 2014;79:191-201.

3. Moss A, Bourke MJ, Hourigan LF, et al. Endoscopic resection for Barrett's high-grade dysplasia and early esophageal adenocarcinoma: an essential staging procedure with long-term therapeutic benefit. Am J Gastroenterol 2010;105:1276-1283.

4. Conio M, Repici A, Cestari R, et al. Endoscopic mucosal resection for high-grade dysplasia and intramucosal carcinoma in Barrett's esophagus: an Italian experience. World J Gastroenterol 2005; 11:6650-6655.

5. Pioche M. Can we really continue to diagnose high grade dysplasia in Barrett's esophagus in Europe without magnified virtual chromo-endoscopy? Endosc Int Open 2015;3:E29-E30.

6. Bertani H, Frazzoni M, Dabizzi E, et al. Improved detection of incident dysplasia by probe-based confocal laser endomicroscopy in a Barrett's esophagus surveillance program. Dig Dis Sci 2013;58:188-193.

7. Bajbouj M, Vieth M, Rösch T, et al. Probe-based confocal laser endomicroscopy compared with standard four-quadrant biopsy for evaluation of neoplasia in Barrett's esophagus. Endoscopy 2010;42:435-440.

8. Canto MI, Anandasabapathy S, Brugge W, et al. In vivo endomicroscopy improves detection of Barrett's esophagus-related neoplasia: a multicenter international randomized controlled trial. Gastrointest Endosc 2014;79:211-221.

9. Sharma P, Meining AR, Coron E, et al. Real-time increased detection of neoplastic tissue in Barrett's esophagus with probe-based confocal laser endomicroscopy: final results of an international multicenter, prospective, randomized, controlled trial. Gastrointest Endosc 2011;74:465-472.

10. Wallace M, Lauwers GY, Chen Y, et al. Miami classification for probe-based confocal laser endomicroscopy. Endoscopy 2011;43:882-891.

11. Gupta A, Attar BM, Koduru P, Murali AR, Go BT, Agarwal R. Utility of confocal laser endomicroscopy in identifying highgrade dysplasia and adenocarcinoma in Barrett's esophagus: a systematic review and meta-analysis. Eur J Gastroenterol Hepatol 2014;26:369-377.

12. Wani S, Abrams J, Edmundowicz SA, et al. Endoscopic mucosal resection results in change of histologic diagnosis in Barrett's esophagus patients with visible and flat neoplasia: a multicenter cohort study. Dig Dis Sci 2013;58:1703-1709.

13. Montgomery E, Bronner MP, Goldblum JR, et al. Reproducibility of the diagnosis of dysplasia in Barrett esophagus: a reaffirmation. Hum Pathol 2001;32:368-378.

14. Bisschops R, Areia M, Coron E, et al. Performance measures for upper gastrointestinal endoscopy: a European Society of Gastrointestinal Endoscopy (ESGE) Quality Improvement 
Initiative. Endoscopy 2016;48:843-864.

15. Maru DM. Barrett's esophagus: diagnostic challenges and recent developments. Ann Diagn Pathol 2009;13:212-221.

16. Downs-Kelly E, Mendelin JE, Bennett AE, et al. Poor interobserver agreement in the distinction of high-grade dysplasia and adenocarcinoma in pretreatment Barrett's esophagus biopsies. Am J Gastroenterol 2008;103:2333-2340.

17. Mino-Kenudson M, Hull MJ, Brown I, et al. EMR for Barrett's esophagus-related superficial neoplasms offers better diagnostic reproducibility than mucosal biopsy. Gastrointest Endosc 2007;66:660-666.

18. Wani S, Mathur SC, Curvers WL, et al. Greater inter observer agreement by endoscopic mucosal resection than biopsy samples in Barrett's dysplasia. Clin Gastroenterol Hepatol 2010;8:783-788.

19. Werbrouck E, De Hertogh G, Sagaert X, et al. Oesophageal biopsies are insufficient to predict final histology after endoscopic resection in early Barrett's neoplasia. United European Gastroenterol J 2016;4:663-668.

20. Pech O, Bollschweiler E, Manner H, Leers J, Ell C, Hölscher AH. Comparison between endoscopic and surgical resection of mucosal esophageal adenocarcinoma in Barrett's esophagus at two highvolume centers. Ann Surg 2011;254:67-72.

21. Tharavej C, Hagen JA, Peters JH, et al. Predictive factors of coexisting cancer in Barrett's high-grade dysplasia. Surg Endosc 2006;20:439-443.

22. Nigro JJ, Hagen JA, DeMeester TR, et al. Occult esophageal adenocarcinoma: extent of disease and implications for effective therapy. Ann Surg 1999;230:433-438.

23. Moss A, Bourke MJ, Hourigan LF, et al. Endoscopic resection for Barrett's high-grade dysplasia and early esophageal adenocarcinoma: an essential staging procedure with long-term therapeutic benefit. Am J Gastroenterol 2010;105:1276-1283.
24. Ahmad NA, Kochman ML, Long WB, Furth EE, Ginsberg GG Efficacy, safety, and clinical outcomes of endoscopic mucosal resection: a study of 101 cases. Gastrointest Endosc 2002;55:390-396.

25. Nijhawan PK, Wang KK. Endoscopic mucosal resection for lesions with endoscopic features suggestive of malignancy and highgrade dysplasia within Barrett's esophagus. Gastrointest Endosc 2000;52:328-332.

26. Seewald S, Akaraviputh T, Seitz U, et al. Circumferential EMR and complete removal of Barrett's epithelium: a new approach to management of Barrett's esophagus containing high-grade intraepithelial neoplasia and intramucosal carcinoma. Gastrointest Endosc 2003;57:854-859.

27. Kariv R, Plesec TP, Goldblum JR, et al. The Seattle protocol does not more reliably predict the detection of cancer at the time of esophagectomy than a less intensive surveillance protocol. Clin Gastroenterol Hepatol 2009;7:653-658.

28. Falk GW, Rice TW, Goldblum JR, Richter JE. Jumbo biopsy forceps protocol still misses unsuspected cancer in Barrett's esophagus with high-grade dysplasia. Gastrointest Endosc 1999;49:170-176.

29. Pohl J, Pech O, May A, Manner H, Fissler-Eckhoff A, Ell C. Incidence of macroscopically occult neoplasias in Barrett's esophagus: are random biopsies dispensable in the era of advanced endoscopic imaging? Am J Gastroenterol 2010;105:2350-2356.

30. Sharma P, Hawes RH, Bansal A, et al. Standard endoscopy with random biopsies versus narrow band imaging targeted biopsies in Barrett's oesophagus: a prospective, international, randomised controlled trial. Gut 2013;62:15-21.

31. Haidry RJ, Butt MA, Dunn JM, et al; UK RFA Registry. Improvement over time in outcomes for patients undergoing endoscopic therapy for Barrett's oesophagus-related neoplasia: 6-year experience from the first 500 patients treated in the UK patient registry. Gut 2015;64:1192-1199. 